\title{
Linear and Nonlinear Effective Medium Properties of Metallodielectric Composites of Interacting Spheres and Isolated Spheroids
}

\author{
Dana C. Kohlgraf-Owens ${ }^{1}$ and Pieter G. Kik ${ }^{1,2}$ \\ ${ }^{I}$ CREOL \&FPCE: The College of Optics and Photonics, University of Central Florida, 4000 Central Florida Blvd, Orlando, FL 32816 \\ ${ }^{2}$ Also with the Department of Physics, University of Central Florida, 4000 Central Florida Blvd, Orlando, FL 32816 \\ Corresponding author email: kohlgraf@creol.ucf.edu, phone: (407) 823-6800, fax: (407) 823-6880
}

\begin{abstract}
We compute the effective medium properties for Kerr-type metallodielectric composites of interacting spheres and isolated spheroids. We show the nonlinear index enhancement increases significantly faster than the linear absorption as particles interact or become elongated.

(C)2008 Optical Society of America

OCIS codes: (050.2065) Effective medium theory, (160.1245) Artificially engineered materials
\end{abstract}

\section{Introduction}

The design of nanostructured materials with properties significantly different from their constituents is a current area of strong interest. One thrust in this area is designing materials with simultaneously high nonlinear (NL) absorption and low linear absorption. A potential candidate to realize this is resonant metal nanoparticles, which produce high local field enhancements, resulting in significantly enhanced nonlinearities. While the field enhancement outside the particles has been extensively studied for sensing applications, comparatively few studies systematically investigate the effect of resonant metal nanoparticles on the metal NL response or composite linear absorption. We use a combination of numerical simulations and effective medium theory to compute the linear and enhancement of the Kerr-type NL effective medium properties in a composite of resonant metal nanoparticles in a dielectric host.

\section{Theory}

The linear and the enhancement of the NL effective medium properties of random distributions of small isotropic spherical nanoparticles embedded in a homogeneous, isotropic host are computed using Eq. 1 and $2[1,2]$.

$$
\begin{gathered}
\varepsilon_{c}=\frac{\left|\int_{i} \varepsilon_{i} \vec{E} d V+\int_{h} \varepsilon_{h} \vec{E} d V\right|}{\left|V_{t}\langle\vec{E}\rangle\right|} \\
g_{h, i}^{(3)} \equiv \frac{\chi_{c}^{(3)}}{\chi_{h, i}^{(3)}}=\frac{\int_{h, i} \vec{E}^{2}|\vec{E}|^{2} d V}{V_{t}\langle\vec{E}\rangle^{2}|\langle\vec{E}\rangle|^{2}}
\end{gathered}
$$

Here $\varepsilon_{\mathrm{c}}, \varepsilon_{\mathrm{i}}$ and $\varepsilon_{\mathrm{h}}$ are the linear dielectric functions in the composite, inclusion and host respectively, $\mathrm{E}$ is the electric field, $V_{t}$ is the total volume of a unit cell, $\chi^{(3)}$ is the third order susceptibility, $g^{(3)}$ is the third-order susceptibility enhancement and $\langle\vec{E}\rangle$ is the volume averaged $\mathrm{E}$ field in the unit cell. For nanoparticles arranged in orthogonal crystal lattices such as cubic or orthorhombic excited with the E-field polarized along a principle axis, here the xaxis, we can use Eq. 1 and 2 to compute $\varepsilon_{\mathrm{c}, \mathrm{x}}$ and $\mathrm{g}_{\mathrm{h} . \mathrm{i}, \mathrm{xxxx}}{ }^{(3)}$. A NL refractive index enhancement $\mathrm{g}_{2}$ can be computed using Eq. 3 where $\eta_{2}$ is the complex NL refractive index and $n$ and $\kappa$ are the real and imaginary parts of the linear refractive index.

$$
g_{2 h, i}=\frac{\eta_{2, c}}{\eta_{2, h, i}}=\frac{\left|\eta_{h, i}\right|^{2}}{\left|\eta_{c}\right|^{2}} \frac{1-i\left(\kappa_{c} / n_{c}\right)}{1-i\left(\kappa_{h, i} / n_{h, i}\right)} g_{h, i}^{(3)}
$$

\section{Methods}

We simulate silver $(\mathrm{Ag})$ nanoparticles embedded in a constant index of refraction of 1.5. For $\mathrm{Ag}$, we use a surface scattering corrected Drude model fit of Johnson and Christy data [3] shown in Eq. 4

$$
\varepsilon_{i}=\varepsilon_{\infty}-\omega_{p}^{2} /\left[\omega^{2}+i\left(\Gamma_{0}+\Gamma_{s}\right) \omega\right]
$$




\section{MThB3.pdf}

where $\varepsilon_{\infty}=5.451, \omega_{p}=1.474 \times 10^{16}[\mathrm{rad} / \mathrm{s}]$, and $\Gamma_{0}$, the fitted bulk electron scattering rate is $8.354 \times 10^{13}\left[\mathrm{~s}^{-1}\right]$. The surface scattering rate, $\Gamma_{s}$, is given by $A v_{f} / r=2.8 \times 10^{14}$, where A is set to $1, v_{f}=1.39 \times 10^{6} \mathrm{~m} / \mathrm{s}$ is the Fermi velocity in silver, and $r$ is the radius of the particle, set to $5 \mathrm{~nm}$ for all simulations.

Simulations are performed in frequency domain using Microwave Studio [4], a finite integration technique code. Tetrahedral meshing is used to realize physically realistic field distributions with minimal mesh grid density. The tangential electric and magnetic fields are set to zero on along the $\mathrm{x}$ and $\mathrm{y}$ boundaries respectively, effectively creating periodic boundary conditions in the $\mathrm{x}$ and $\mathrm{y}$ directions. The effective medium properties are then computed using Eq. 1-3. Numerical convergence tests indicate that the linear properties and $\mathrm{g}_{\mathrm{h}, \mathrm{xxxx}}{ }^{(3)}$ have an error $<2 \%$ and that $\mathrm{g}_{\mathrm{i}, \mathrm{xxxx}}{ }^{(3)}$ values are within $10 \%$ of the fully converged result for all simulations discussed here.

\section{Results and Discussion}

\subsection{Interacting Spheres}

First we consider the effect of varying the particle separation in an orthorhombic lattice of particles with varying $\mathrm{x}$ and y spacing. The unit cell is $30 \mathrm{~nm}$ long along the direction of propagation and contains a $10 \mathrm{~nm}$ diameter Ag sphere in the center. For a square lattice the unit cell is $24 \mathrm{~nm}$ in length ( $\mathrm{dx}$ ) and width (dy), corresponding to a volume fill fraction of 0.03 . For the case where $d x=d y=24 \mathrm{~nm}$, interparticle interactions were found to be small (no significant change of the plasmon resonance relative to that of isolated particles). To vary interparticle interactions, $\mathrm{dx}$ or dy is set to 12.5 or $15 \mathrm{~nm}$ while dy or $\mathrm{dx}$ is adjusted to maintain the same unit cell volume for all simulations.

The inset in Fig. 1(b) displays the composite linear absorption, showing strong absorption due to the nanoparticle plasmon resonance. The plasmon resonance peak red-shifts and increases $\sim 15 \%$ as the particle separation is reduced from $24 \mathrm{~nm}$ to 12.5 along the x-direction. The peak absorption coefficients correspond to 1/e depths between 50 and $65 \mathrm{~nm}$. Figure 1(a) shows the enhancement of the NL refractive index in the host. This factor is complex, indicating that coupling exists between the real and imaginary parts of the host and composite refractive index. One physically intuitive way to understand this is to consider the effect of changes in the host refractive index on the location and strength of the plasmon resonance [5]. Specifically, a small change in the host or inclusion refractive index causes a shift in the location of the plasmon resonance, whereas a small change in the host or inclusion absorption affects the strength of the plasmon resonance. These effects influence both the real and imaginary parts of the composite response, which can be mathematically expressed in terms of a complex enhancement factor.
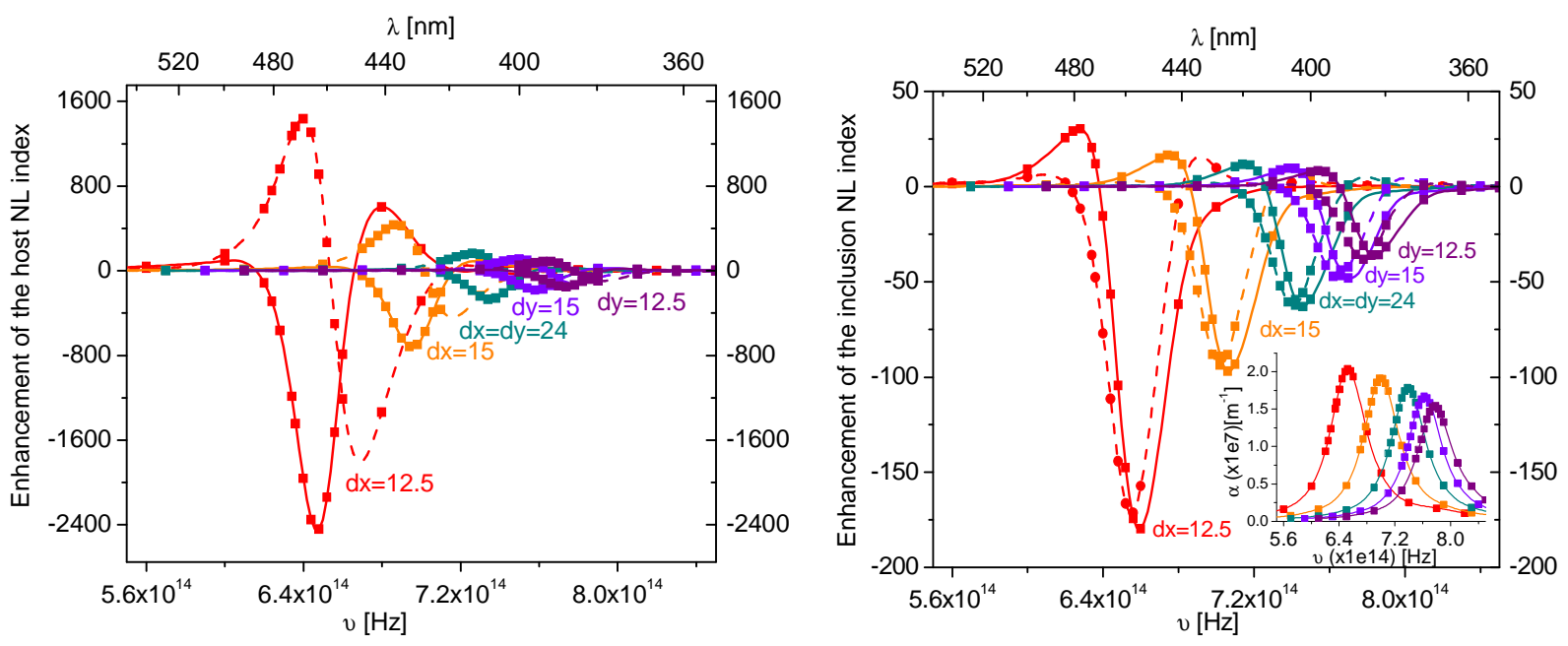

Fig. 1: (a) Enhancement of the host nonlinear index for different interparticle separations. (b) Enhancement of the inclusion nonlinear index. Inset: Composite linear absorption. Solid and dashed lines represent spline fits to the real and imaginary parts of the enhancement factor.

Figure 1(a) shows that the peak NL index enhancement increases as the interparticle separation decreases along the $\mathrm{x}$-axis, with an order of magnitude increase occurring when the center to center particle separation is reduced from $24 \mathrm{~nm}$ to $12.5 \mathrm{~nm}$ along the direction of polarization. This increase can be largely attributed to the "focusing" of near field radiation between particles as they are brought closer together along the x-direction. The enhancement of the inclusion complex NL refractive index is shown in Fig. 1(b). Decreasing the interparticle separation along the $\mathrm{X}$-axis results in a factor of 3 improvement in the peak NL index enhancement as the interparticle separation is 


\section{MThB3.pdf}

reduced from $24 \mathrm{~nm}$ to $12.5 \mathrm{~nm}$. This increase predominately results from the significant increase in $\kappa_{\mathrm{i}} / \mathrm{n}_{\mathrm{i}}$ in Eq. 3 as the frequency is reduced. From these results we find the peak enhancement of the host and inclusion nonlinearity respectively increases 65 and 20 times faster than the linear absorption as dx is reduced from $24 \mathrm{~nm}$ to $12.5 \mathrm{~nm}$.

\subsection{Isolated Spheroids of Varying Aspect Ratio}

The second geometry we discuss is isolated spheroids of varying aspect ratio (AR). The initial unit cell consists of a $10 \mathrm{~nm}$ diameter Ag spherical nanoparticle centered in a $28 \mathrm{~nm}$ cubic cell, resulting in a fill fraction of $\sim 0.024$. Prolate spheroids of different AR are created and the sides of the unit cell are adjusted to minimize interparticle interactions. Both the unit cell and particle volumes are held constant for all simulations.

Figures 2(a) and (b) respectively show the index enhancement factor for the host and inclusion nonlinearity. We observe the same trends in the enhancement of the NL refractive index with increasing AR as for decreasing particle separation shown in Fig. 1, with a factor 3.5 and 7 increase in the NL index enhancement of the host and inclusions respectively as the $\mathrm{AR}$ increases from 1 to 3 . The inset in Fig. 2(b) shows the composite linear absorption, which increases by $\sim 50 \%$ as the AR is increased from 1 to 3 . In this case, the enhancement of the host and inclusion nonlinearity is found to increase respectively 7 and 14 times faster than the linear absorption.
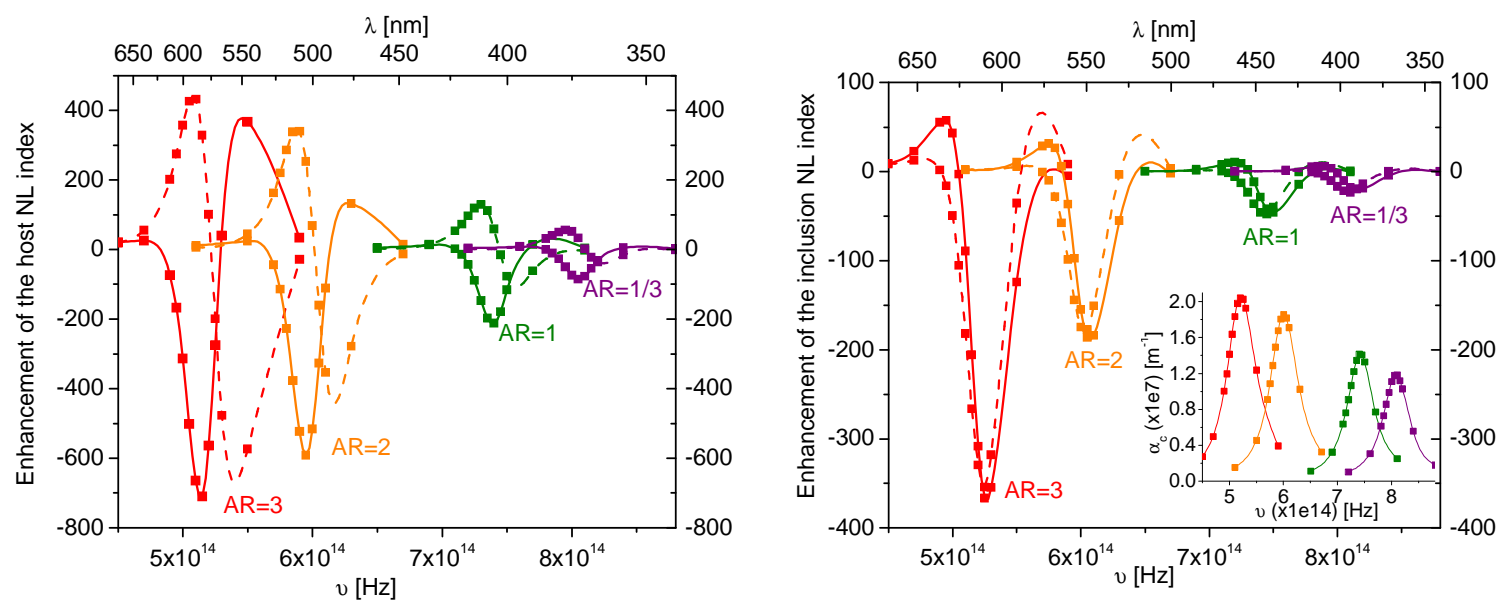

Fig 2: (a) Enhancement of the host nonlinear refractive index for isolated prolate spheroids of different aspect ratios. (b) Enhancement of the inclusion nonlinear refractive index. Inset: Composite linear absorption. Solid and dashed lines represent spline fits to the real and imaginary parts of the enhancement factor respectively.

\section{Summary and Conclusions}

The composite linear absorption and the enhancement of the host and inclusion NL refractive index are calculated for orthorhombic lattices of resonant metal nanoparticles embedded in a dielectric host. For spherical nanoparticles the peak enhancement of the host and inclusion NL refractive index increases 65 and 20 times faster than the linear absorption as the interparticle separation is reduced from 24 to $12.5 \mathrm{~nm}$ along the x-direction. For isolated spheroids, the peak host and inclusion NL index enhancement increases 7 and 14 times faster than the linear absorption as the AR is increased from 1 to 3. This significant improvement of the NL index enhancement relative to the linear absorption may have implications in the design of NL optical devices.

\section{References}

[1] M. I. Stockman, K. B. Kurlayev, and T. F. George, "Linear and nonlinear optical susceptibilities of Maxwell Garnett composites: Dipolar spectral theory," Physical Review B 60, 17071-17083 (1999).

[2] J. E. Sipe and R. W. Boyd, "Nonlinear Susceptibility of Composite Optical-Materials in the Maxwell Garnett Model," Physical Review A 46, 1614-1629 (1992).

[3] P. B. Johnson and R. W. Christy, "Optical-Constants Of Noble-Metals," Physical Review B 6, 4370-4379 (1972).

[4] G. f. Computer-Simulationstechnik, Microwave Studio (MWS), Darmstadt, Germany, 2008.

[5] D. C. Kohlgraf-Owens and P. G. Kik, "Numerical study of surface plasmon enhanced nonlinear absorption and refraction," submitted to Optics Express (2008). 